\title{
THE GENERALIZED HOMOLOGY OF PRODUCTS
}

\author{
MARK HOVEY \\ Department of Mathematics, Wesleyan University, Middletown, CT 06459 \\ e-mail:hovey@member.ams.org
}

(Received 1 January, 2005; accepted 18 September, 2006)

\begin{abstract}
We construct a spectral sequence that computes the generalized homology $E_{*}\left(\prod X^{\alpha}\right)$ of a product of spectra. The $E_{2}$-term of this spectral sequence consists of the right derived functors of product in the category of $E_{*} E$-comodules, and the spectral sequence always converges when $E$ is the Johnson-Wilson theory $E(n)$ and the $X^{\alpha}$ are $L_{n}$-local. We are able to prove some results about the $E_{2}$-term of this spectral sequence; in particular, we show that the $E(n)$-homology of a product of $E(n)$-module spectra $X^{\alpha}$ is just the comodule product of the $E(n)_{*} X^{\alpha}$. This spectral sequence is relevant to the chromatic splitting conjecture.
\end{abstract}

2000 Mathematics Subject Classification. 55T25, 55N22, 55P60, 18G10, 16W30.

Introduction. The basic tools of computation in algebraic topology are homology theories. Homology theories preserve coproducts, but can behave very badly on products. There are examples of homology theories $E$ and sets of spectra (generalized spaces) $\left\{X^{\alpha}\right\}$, for which $E_{*} X^{\alpha}=0$ for all $i$ and yet $E_{*}\left(\prod_{\alpha} X^{\alpha}\right) \neq 0$. Indeed, we can take $E=H \mathbb{Q}$, rational homology, where we have $(H \mathbb{Q})_{*}\left(H \mathbb{Z} / p^{k}\right)=0$ for all $k$, but

$$
(H \mathbb{Q})_{*}\left(\prod_{k} H \mathbb{Z} / p^{k}\right)=\left(\prod_{k} \mathbb{Z} / p^{k}\right) \otimes \mathbb{Q} \neq 0
$$

since, for example, the element $(1,1,1, \ldots)$ is not torsion.

Despite this counterexample, in this paper we build a spectral sequence that converges to $E_{*}\left(\prod X^{\alpha}\right)$ in good cases. The most important good case is when $E=E(n)$, the Johnson-Wilson theory of great importance in stable homotopy theory. The $E_{2}$ term of this spectral sequence is made up of the right derived functors of product applied to $\left\{E_{*} X^{\alpha}\right\}$. Of course, the product is exact in the category of $E_{*}$-modules, so these derived functors are instead taken in the category of $E_{*} E$-comodules, where products remain mysterious.

The usefulness of this spectral sequence will depend on our knowledge of its $E_{2}$-term. At this point, the author knows very little about the derived functors $\prod_{E(n)_{*} E(n)}^{s} M^{\alpha}$ of product in the category of $E(n)_{*} E(n)$-comodules. The most important conjecture about them is that $\prod_{E(n)_{*} E(n)}^{s} M^{\alpha}=0$ for all $s \geq N$ for some $N$, so that the spectral sequence has a horizontal vanishing line at the $E_{2}$ term (we show that the spectral sequence does have a horizontal vanishing line at some $E_{r}$ term). We expect that $N$ is very close to $n$ itself.

We do prove that derived functors of product can be computed using relatively injective resolutions, such as the cobar complex, rather than honest injective resolutions. 
It follows that

$$
E(n)_{*}\left(\prod X^{\alpha}\right) \cong \prod_{E(n)_{*} E(n)} E(n)_{*} X^{\alpha}
$$

for a family of $E(n)$-module spectra $X^{\alpha}$. We also construct a spectral sequence relating derived functors of product in the category of $E(n)_{*} E(n)$-comodules to derived functors of product in the category of $B P_{*} B P$-comodules. The category of $B P_{*} B P$-comodules is easier to cope with since $B P_{*} B P$ is connective and free over $B P_{*}$. These results give the author hope that these derived functors will be understood at some point, though at the moment he does not even understand them in the simple case of $E(1)_{*} E(1)$-comodules.

The reason for the author's interest in this spectral sequence is the chromatic splitting conjecture [5] of Mike Hopkins. Recall that the simplest form of the chromatic splitting conjecture is that $K(n-1)_{*} L_{K(n)} X$ is a direct sum of two copies of $K(n-1)_{*} X$, for $X$ a finite $p$-complete spectrum. Also recall that $L_{K(n)} X$ is a homotopy inverse limit $\operatorname{holim}_{I}\left(L_{n} X \wedge S / I\right)$ analogous to completion at the ideal $\left(p, v_{1}, \ldots, v_{n-1}\right)$. This result is due to Hopkins; a precise statement of it can be found in [8, Proposition 7.10]. Therefore, if one has a spectral sequence for the $E(n-1)$-homology of a homotopy inverse limit, one might be able to compute $E(n-1)_{*}\left(L_{K(n)} X\right)$ and therefore $K(n-1)_{*}\left(L_{K(n)} X\right)$.

This approach to the chromatic splitting conjecture is due to Mike Hopkins, and is based on the work of Paul Goerss [4], who constructed a spectral sequence for the $\bmod p$ homology of a homotopy inverse limit of spaces. Hopkins suggested this idea to Hal Sadofsky and the author after a talk by Goerss. Sadofsky has constructed a spectral sequence for the $E(n)$-homology of a homotopy inverse limit, as envisioned by Hopkins, and has proved some results about it that are relevant to the chromatic splitting conjecture. Unfortunately, Sadofsky has not yet made a preprint of his work available.

The author decided instead to begin with the simpler case of products, though the methods used in this paper can also be used to construct a version of Sadofsky's spectral sequence. To the author's knowledge, Sadofsky has not considered products. But the author acknowledges his heavy debt to the work of Sadofsky. He also would like to thank Mike Hopkins for his original suggestion, and Paul Goerss for his paper [4], without which this paper would never have been written.

1. The modified Adams tower. The first step in constructing a spectral sequence is to resolve the object one is considering. In our case, the resolution we need is called the modified Adams tower and is due to Devinatz and Hopkins [3]. The idea is to mimic the usual construction of an injective resolution using $E_{*}$-injectives, where $E$ is a well-behaved homology theory. We will have to assume that $E$ is a commutative ring spectrum such that $E_{*} E$ is flat over $E_{*}$; it is well-known [11, Proposition 2.2.8] that this implies that $\left(E_{*}, E_{*} E\right)$ is a flat Hopf algebroid and that $E_{*} X$ is naturally a left $E_{*} E$-comodule for a spectrum $X$. It also implies that $E_{*} E$-comodules form an abelian category [11, Theorem A1.1.3] with enough injectives [11, Lemma A1.2.2].

The following definition is taken from [3].

Definition 1.1. Let $E$ be a commutative ring spectrum such that $E_{*} E$ is flat over $E_{*}$. Define a functor $D$ from injective $E_{*} E$-comodules to the stable homotopy category $\mathcal{S}$ as follows. Given an injective $E_{*} E$-comodule $I$, consider the functor $D_{I}$ from spectra 
to abelian groups defined by

$$
D_{I}(X)=\operatorname{Hom}_{E_{*} E}\left(E_{*} X, I\right) .
$$

Then $D_{I}$ is a cohomology functor, so there is a unique spectrum $D(I)$ such that there is a natural isomorphism

$$
D_{I}(X) \cong[X, D(I)]
$$

The hypotheses we have given on $E$ are sufficient to define $D(I)$, but apparently insufficient to compute $E_{*} D(I)$. For this we need some form of the following definition; this particular form comes from [6].

DEFINITION 1.2. A ring spectrum $E$ is called topologically flat if $E$ is the minimal weak colimit of a filtered diagram of finite spectra $X_{i}$ such that $E_{*} X_{i}$ is a finitely generated projective $E_{*}$-module.

Minimal weak colimits are discussed in [7, Section 2.2]. Adams [1, Section III.13] proves that many standard spectra such as $B P$ are topologically flat; in addition, any Landweber exact commutative ring spectrum over $B P$ or $M U$ is topologically flat [6, Theorem 1.4.9]. Note that if $E$ is topologically flat, then $E_{*} E$ is flat over $E_{*}$, since it is the colimit of projective modules.

The following theorem is a translation of Theorem 1.5 of [3] to this terminology.

THEOREM 1.3. Suppose $E$ is a topologically flat commutative ring spectrum, and $I$ is an injective $E_{*} E$-comodule. Then there is a natural isomorphism $E_{*} D(I) \cong I$.

We can now describe the modified Adams tower. Let $E$ be a topologically flat commutative ring spectrum, and suppose we have a spectrum $X$. Let $C=E_{*} X$, and choose an injective resolution

$$
0 \rightarrow C \stackrel{\eta}{\rightarrow} I_{0} \stackrel{\tau_{0}}{\rightarrow} I_{1} \stackrel{\tau_{1}}{\rightarrow} \cdots
$$

of $C$ in the category of $E_{*} E$-comodules. Let $\eta_{s}: C_{s} \rightarrow I_{s}$ denote the kernel of $\tau_{s}$, so that $\eta_{0}=\eta$.

As explained in [3, Section 1], we can use this resolution of $C$ to build a tower over $X$ with good properties. More precisely, we have the following lemma, which is easily proved by induction on $n$.

LEMMA 1.4. Let $E$ be a topologically flat commutative ring spectrum, let $X$ be a spectrum, and choose an injective resolution of $E_{*} X$ as above. Then there is a tower

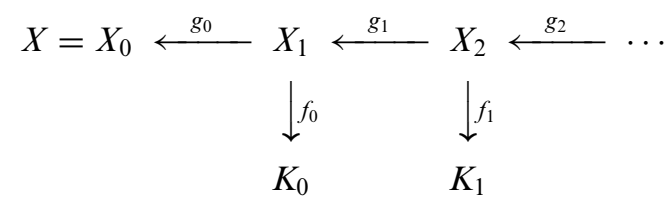

over $X$ satisfying the following properties.

(a) $K_{s}=\Sigma^{-s} D\left(I_{s}\right)$.

(b) $X_{s+1}$ is the fiber of $f_{s}$.

(c) $E_{*} X_{s} \cong \Sigma^{-s} C_{s}$.

(d) The map $f_{s}$ is induced by the inclusion $C_{s} \rightarrow I_{s}$. 
(e) $E_{*} g_{s}=0$, and the boundary map $K_{s} \rightarrow \Sigma X_{s+1}$ induces the surjection $\Sigma^{-s} I_{s} \rightarrow$ $\Sigma^{-s} C_{s+1}$ on $E_{*}$-homology.

We call this tower the modified Adams tower for $X$ based on $E$-homology. Of course, it actually depends on the injective resolution as well. We obtain a spectral sequence by applying $[Z,-]$ for any $Z$ to get the modified Adams spectral sequence of Devinatz [3]; its $E_{2}$-term is $\operatorname{Ext}_{E_{*} E}^{* *}\left(E_{*} X, E_{*} Y\right)$, it is independent of the choice of resolution from the $E_{2}$ page on, and in good cases it converges to $\left[Z, L_{E} X\right]_{*}$.

2. Products of comodules. In order to understand the spectral sequence for products of spectra, we need to know a little about products of comodules. So suppose $(A, \Gamma)$ is a flat Hopf algebroid. As mentioned above, basic facts about the category of $\Gamma$-comodules can be found in [11, Appendix 1], though he does not discuss products. A more in-depth look at the global structure of the category of $\Gamma$-comodules, including products, can be found in [6].

The main point of interest here is that the forgetful functor to $A$-modules does not preserve products. It is easiest to understand this when $\Gamma$ is free over $A$. In this case, every element $m$ in a $\Gamma$-comodule $M$ has a diagonal of the form $\sum \gamma_{i} \otimes m_{i}$, where $\gamma_{i}$ runs through a basis of $\Gamma$ as a right $A$-module, and all but finitely many of the $m_{i}$ are zero. In the $A$-module product $\prod_{\alpha} M^{\alpha}$ of comodules $M^{\alpha}$, there may well be elements whose diagonal would have to be infinitely long. In fact, when $\Gamma$ is projective over $A$, the comodule product $\prod_{\Gamma} M^{\alpha}$ is the submodule of $\prod M^{\alpha}$ consisting of those elements whose diagonal lands in

$$
\Gamma \otimes_{A} \prod M^{\alpha} \subseteq \prod\left(\Gamma \otimes_{A} M^{\alpha}\right) .
$$

To construct the product when $\Gamma$ is only assumed to be flat over $A$, one first checks that

$$
\prod_{\Gamma}\left(\Gamma \otimes_{A} N^{\alpha}\right) \cong \Gamma \otimes_{A}\left(\prod N^{\alpha}\right)
$$

for $A$-modules $N^{\alpha}$, where $\Gamma \otimes_{A} P$ denotes the extended $\Gamma$-comodule, in which $\Gamma$ coacts only on the $\Gamma$ factor. One then constructs $\prod_{\Gamma} f^{\alpha}$, where $f^{\alpha}$ is an arbitrary map of extended comodules. Finally, given arbitrary comodules $M^{\alpha}$, we have exact sequences of comodules

$$
0 \rightarrow M^{\alpha} \stackrel{\psi}{\rightarrow} \Gamma \otimes_{A} M^{\alpha} \stackrel{f^{\alpha}}{\rightarrow} \Gamma \otimes_{A} N^{\alpha}
$$

where $N^{\alpha}$ is the cokernel of $\psi$, and $f^{\alpha}$ is the composite

$$
\Gamma \otimes_{A} M^{\alpha} \rightarrow N^{\alpha} \stackrel{\psi}{\rightarrow} \Gamma \otimes_{A} N^{\alpha} .
$$

It follows that $\prod_{\Gamma} M^{\alpha} \cong \operatorname{ker} \prod_{\Gamma} f^{\alpha}$. Details can be found in [6].

This construction shows that the product of comodules is more complicated than one would want; in particular, it is not always exact (see the example before Proposition 1.2.3 of [6]). As a right adjoint, of course, the product is left exact. Since there are enough injective $\Gamma$-comodules, the product will have right derived functors $\prod_{\Gamma}^{s} M^{\alpha}$ for $s \geq 0$. Almost nothing is known about these right derived functors, but they are what will appear as the $E_{2}$-term in our spectral sequence. 
For the construction of our spectral sequence, we need the following proposition.

Proposition 2.1. Suppose $E$ is a topologically flat commutative ring spectrum, and $\left\{I_{\alpha}\right\}$ is a family of injective $E_{*} E$-comodules. Then there is a natural isomorphism

$$
D\left(\prod_{E_{*} E} I_{\alpha}\right) \rightarrow \prod D\left(I_{\alpha}\right) .
$$

Here the notation $\prod_{E_{*} E}$ denotes the product in the category of $E_{*} E$-comodules.

Proof. Note that $\prod_{E_{*} E} I_{\alpha}$ is again an injective comodule. The functoriality of $D$ guarantees the existence of this map. Now, if $X$ is an arbitrary spectrum, we have a chain of isomorphisms

$$
\begin{aligned}
{\left[X, D\left(\prod_{E_{*} E} I_{\alpha}\right)\right] } & \cong \operatorname{Hom}_{E_{*} E}\left(E_{*} X, \prod_{E_{*} E} I_{\alpha}\right) \cong \prod \operatorname{Hom}_{E_{*} E}\left(E_{*} X, I_{\alpha}\right) \\
& \cong \prod\left[X, D\left(I_{\alpha}\right)\right] \cong\left[X, \prod D\left(I_{\alpha}\right)\right] .
\end{aligned}
$$

This gives us the desired isomorphism.

3. Construction of the spectral sequence. We can now use the modified Adams towers of Lemma 1.4 to construct our spectral sequence.

THEOREM 3.1. Let E be a topologically flat commutative ring spectrum, and let $\left\{X^{\alpha}\right\}$ be a family of spectra. There is a natural spectral sequence $E_{*}^{* *}\left(\left\{X^{\alpha}\right\}\right)$ with $d_{r}^{s, t}: E_{r}^{s, t} \rightarrow$ $E_{r}^{s+r, t+r-1}$ and $E_{2}$-term

$$
E_{2}^{s, t} \cong\left(\prod_{E_{*} E}^{s} E_{*} X^{\alpha}\right)_{t}
$$

This is a spectral sequence of $E_{*} E$-comodules, in the sense that each $d_{r}^{s, *}$ is a map of $E_{*} E$ comodules of degree $r-1$. Furthermore, every element in $E_{2}^{0, t}$ in the image of the natural map

$$
\bigoplus E_{*} X^{\alpha} \rightarrow \prod_{E_{*} E} E_{*} X^{\alpha}
$$

is a permanent cycle.

Proof. We have modified Adams towers $X_{s}^{\alpha}$ for each $X^{\alpha}$. Taking the product gives us the tower below.

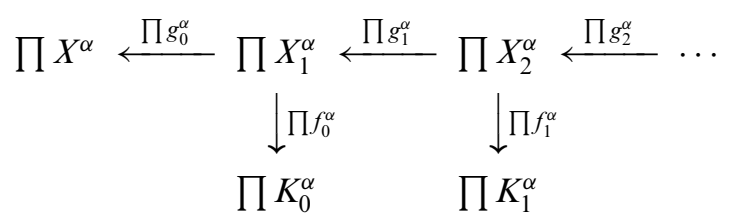

By applying $E_{*}$-homology, we get an associated exact couple and spectral sequence. That is, we let $D_{1}^{s, t}=E_{t-s}\left(\prod X_{s}^{\alpha}\right)$ and $E_{1}^{s, t}=E_{t-s}\left(\prod K_{s}^{\alpha}\right)$. We define $i_{1}: D \rightarrow D$ of bidegree $(-1,-1)$ by $i_{1}^{s, t}=E_{t-s}\left(\prod g_{s}^{\alpha}\right)$, we define $j_{1}: D \rightarrow E$ of bidegree $(0,0)$ by $j_{1}^{s, t}=E_{t-s}\left(\prod f_{s}^{\alpha}\right)$, and we define $k_{1}: E \rightarrow D$ of bidegree $(1,0)$ in bidegree $(s, t)$ to be $E_{t-s}$ of the boundary map $\prod K_{s}^{\alpha} \rightarrow \Sigma \prod X_{s+1}^{\alpha}$. All of these maps are maps of comodules, 
and therefore the resulting spectral sequence will be a spectral sequence of comodules, as claimed.

By combining Proposition 2.1 with Theorem 1.3, we see that

$$
E_{1}^{s, t} \cong E_{t-s}\left(\prod \Sigma^{-s} D\left(I_{s}^{\alpha}\right)\right) \cong E_{t} D\left(\prod_{E_{*} E} I_{s}^{\alpha}\right) \cong\left(\prod_{E_{*} E} I_{s}^{\alpha}\right)_{t}
$$

One can easily check that the first differential $d_{1}$ is $\prod_{E_{*} E} \tau_{s}^{\alpha}$, and therefore that the $E_{2}$-term is as claimed.

Naturality now follows in the usual way; a collection of maps $X^{\alpha} \rightarrow Y^{\alpha}$ induces non-canonical maps of the injective resolutions in question, and hence the modified Adams towers. Taking products gives us a map of spectral sequences, which is canonical from $E_{2}$ onwards.

Finally, we can also construct a spectral sequence by taking the wedge of the modified Adams towers of the $X^{\alpha}$ and applying $E_{*}$ homology. This gives a spectral sequence with $D_{1}^{s, t}=\left(\bigoplus C_{s}^{\alpha}\right)_{t}$ and $E_{1}^{s, t}=\left(\bigoplus I_{s}^{\alpha}\right)_{t}$. The $d_{1}$ differential is the obvious one, and so $E_{2}^{0, *} \cong \bigoplus E_{*} X^{\alpha}$ and $E_{2}^{s, t}=0$ for $s>0$. There is a map from the spectral sequence to the spectral sequence for the product of the $X^{\alpha}$. Anything in the image of this map of spectral sequences must be a permanent cycle.

4. Convergence of the spectral sequence. We now discuss the convergence of our spectral sequence. This is a delicate question, in general, as the example given at the beginning of the paper shows. However, the spectral sequence always converges when $E=E(n)$ and each $X^{\alpha}$ is $E(n)$-local.

THEOREM 4.1. Suppose $E=E(n)$ and each $X^{\alpha}$ is $L_{n}$-local. Then the spectral sequence of Theorem 3.1 converges strongly to $E(n)_{*}\left(\prod X^{\alpha}\right)$. Furthermore, it has a horizontal vanishing line at some $E_{r}$ term.

Proof. First note that each $X_{s}^{\alpha}$ is $L_{n}$-local, since $K_{s}^{\alpha}=\Sigma^{-s} D\left(I_{s}^{\alpha}\right)$ is clearly $L_{n}$-local. Each map $g_{s}^{\alpha}: X_{s+1}^{\alpha} \rightarrow X_{s}^{\alpha}$ has $E(n)_{*}\left(g_{s}^{\alpha}\right)=0$. It follows from [10, Theorem 5.10] that there is an $N$, depending on $n$ but independent of $\alpha$, such that each $N$-fold composite $X_{s+N}^{\alpha} \rightarrow X_{s}^{\alpha}$ is null. Hence each composite $\prod X_{s+N}^{\alpha} \rightarrow \prod X_{s}^{\alpha}$ is null, giving us our desired horizontal vanishing line. Hence

$$
\lim _{s} E(n)_{*}\left(\prod X_{s}^{\alpha}\right)=\lim _{s}^{1} E(n)_{*}\left(\prod X_{s}^{\alpha}\right)=0
$$

so the spectral sequence converges conditionally to $E(n)_{*}\left(\prod X^{\alpha}\right)$ [2]. It is also clear that $\lim _{r}^{1} E_{r}^{s, t}=0$, and so the spectral sequence converges strongly as well [2, Theorem 7.3].

5. Relatively injective resolutions and an application. Although we cannot prove very much about the derived functors of products, we can at least show that one can use relatively injective comodules to compute them. This allows us to compute the $E(n)$-homology of products of $E(n)$-module spectra.

Proposition 5.1. Let $(A, \Gamma)$ be a flat Hopf algebroid, and suppose $M^{\alpha}$ is a relatively injective $\Gamma$-comodule for all $\alpha$. Then $\prod_{\Gamma}^{s} M^{\alpha}=0$ for $s>0$. 
Proof. Since $M^{\alpha}$ is relatively injective, it is a retract of $\Gamma \otimes_{A} M^{\alpha}$. It therefore suffices to show that $\prod_{\Gamma}^{s}\left(\Gamma \otimes_{A} M^{\alpha}\right)=0$ for all $s>0$. To do so, choose an injective resolution $I_{*}^{\alpha}$ of $M^{\alpha}$ in the category of $A$-modules. Since $\Gamma$ is flat over $A, \Gamma \otimes_{A} I_{*}^{\alpha}$ is a resolution of $\Gamma \otimes_{A} M^{\alpha}$ in the category of $\Gamma$-comodules. Furthermore, each $\Gamma \otimes_{A} I_{s}^{\alpha}$ is an injective $\Gamma$-comodule [11, Lemma A1.2.2]. Hence

$$
\prod_{\Gamma}^{s}\left(\Gamma \otimes_{A} M^{\alpha}\right) \cong H^{s}\left(\prod_{\Gamma}\left(\Gamma \otimes_{A} I_{*}^{\alpha}\right)\right) \cong H^{s}\left(\Gamma \otimes_{A}\left(\prod I_{*}^{\alpha}\right)\right) .
$$

Since products are exact on the category of $A$-modules, and since $\Gamma$ is flat, these groups are 0 for $s>0$.

This yields an immediate topological corollary.

Corollary 5.2. Suppose $X^{\alpha}$ is an $E(n)$-module spectrum for all $\alpha$. Then

$$
E(n)_{*}\left(\prod X^{\alpha}\right) \cong \prod_{E(n)_{*} E(n)} E(n)_{*}\left(X^{\alpha}\right) .
$$

In particular,

$$
E(n)_{*}\left(\prod E(n) \wedge X^{\alpha}\right) \cong E(n)_{*} E(n) \otimes_{E(n)_{*}}\left(\prod E(n)_{*} X^{\alpha}\right) .
$$

Proof. Since $X^{\alpha}$ is an $E(n)$-module spectrum, it is $L_{n}$-local. Furthermore, $E(n)_{*} X^{\alpha}$ is a retract of

$$
E(n)_{*}\left(E(n) \wedge X^{\alpha}\right) \cong E(n)_{*} E(n) \otimes_{E(n)_{*}} E(n)_{*} X^{\alpha},
$$

so is relatively injective. Proposition 5.1 then implies that the $E_{2}$-term of our spectral sequence is 0 except in bidegree $(0, t)$. It therefore collapses, and we get the desired isomorphism.

It also follows, using standard homological algebra, that we can use relatively injective resolutions to compute the derived functors of product. For example, we can use the cobar resolution $C^{*}(M)$ described in [11, Definition A1.2.10].

Corollary 5.3. Suppose $(A, \Gamma)$ is a flat Hopf algebroid, and $\left\{M^{\alpha}\right\}$ is a set of $\Gamma$-comodules. Let $C^{*}\left(M^{\alpha}\right)$ denote the cobar resolution on $M^{\alpha}$. Then

$$
\prod_{\Gamma}^{s} M^{\alpha} \cong H^{s} \prod_{\Gamma} C^{*} M^{\alpha} .
$$

This corollary tells us, for example, that if $J M^{\alpha}=0$ for some invariant ideal $J$ and all $\alpha$, then $J \prod_{\Gamma}^{s} M^{\alpha}=0$ for all $s$.

6. $\boldsymbol{B} \boldsymbol{P}_{*}$ BP-comodules and $\boldsymbol{E}(\boldsymbol{n})_{*} \boldsymbol{E}(\boldsymbol{n})$-comodules. In this section, we exploit the close relationship between $B P_{*} B P$-comodules and $E(n)_{*} E(n)$-comodules studied in [9] to get some partial understanding of the product of comodules.

We begin with $B P_{*} B P$-comodules, which are easier to handle because $B P_{*} B P$ is connective and projective over $B P_{*}$. As mentioned in Section 2, the product of a family $\left\{M^{\alpha}\right\}$ of $B P_{*} B P$-comodules is the submodule of $\prod M^{\alpha}$ consisting of those elements whose diagonal has finite length.

Definition 6.1. A family of $B P_{*} B P$-comodules $\left\{M^{\alpha}\right\}$ is uniformly bounded below if there is a $d \in \mathbb{Z}$ such that $M_{n}^{\alpha}=0$ for all $n<d$ and all $\alpha$. 
The product and its derived functors are particularly simple for a uniformly bounded below family.

Theorem 6.2. Suppose $(A, \Gamma)=\left(B P_{*}, B P_{*} B P\right)$, and $\left\{M^{\alpha}\right\}$ is a family of $\Gamma$ comodules that is uniformly bounded below. Then

$$
\prod_{\Gamma} M^{\alpha} \cong \prod M^{\alpha} \text { and } \prod_{\Gamma}^{s} M^{\alpha}=0 \text { for all } s>0 .
$$

Proof. Since every element of $\prod M^{\alpha}$ must have finite diagonal, the first statement is clear. For the second statement, consider the cobar resolution $C_{*} M^{\alpha}$ of $M^{\alpha}$ by relatively injective comodules. We have $C_{S} M^{\alpha}=\Gamma \otimes_{A} \bar{\Gamma}^{\otimes s} \otimes_{A} M^{\alpha}$, so, since $\Gamma$ is connective, the family $\left\{C_{s} M^{\alpha}\right\}$ is uniformly bounded below for each $s$. We therefore have

$$
\prod_{\Gamma}^{s} M^{\alpha} \cong H^{s} \prod_{\Gamma} C_{*} M^{\alpha} \cong H^{s} \prod C_{*} M^{\alpha}=0
$$

for $s>0$, using Corollary 5.3 and the fact that products of modules are exact.

To relate this to $E(n)_{*} E(n)$-comodules, we recall from [9] and [10] the exact functor $\Phi_{*}$ from $B P_{*} B P$-comodules to $E(n)_{*} E(n)$-comodules defined by $\Phi_{*} M=E(n)_{*} \otimes_{B P_{*}} M$. The functor $\Phi_{*}$ has a fully faithful right adjoint $\Phi^{*}$, the composite $\Phi_{*} \Phi^{*}$ is naturally isomorphic to the identity, and the composite $L_{n}=\Phi^{*} \Phi_{*}$ is the localization functor on the category of $B P_{*} B P$-comodules with respect to the hereditary torsion theory of $v_{n}$-torsion comodules. The functor $L_{n}$ is left exact, but has right derived functors $L_{n}^{q}$ for $0 \leq q \leq n$, studied in [10].

As a left adjoint, we do not expect $\Phi_{*}$ to preserve products. We do, however, have the following result.

TheOREM 6.3. Suppose $\left\{M^{\alpha}\right\}$ is a family of $B P_{*} B P$-comodules. Then there is a natural isomorphism

$$
\prod_{E(n)_{*} E(n)} \Phi_{*} M^{\alpha} \rightarrow \Phi_{*}\left(\prod_{B P_{*} B P} L_{n} M^{\alpha}\right)
$$

In fact, there is a convergent first quadrant spectral sequence $E_{r}^{p, q}$ of $E(n)_{*} E(n)$-comodules with

$$
E_{2}^{p, q} \cong \Phi_{*}\left(\prod_{B P_{*} B P}^{p}\left(L_{n}^{q} M^{\alpha}\right)\right) \Rightarrow \prod_{E(n)_{*} E(n)}^{p+q} \Phi_{*} M^{\alpha} .
$$

Proof. Since $\Phi^{*}$ is a right adjoint, we have

$$
\prod_{E(n)_{*} E(n)} \Phi_{*} M^{\alpha} \cong \Phi_{*} \Phi^{*}\left(\prod_{E(n)_{*} E(n)} \Phi_{*} M^{\alpha}\right) \cong \Phi_{*} \prod_{B P_{*} B P}\left(L_{n} M^{\alpha}\right),
$$

as required. The spectral sequence is the Grothendieck spectral sequence for the derived functors of the composition, described in [12, Section 5.8]. Recall that this spectral sequence has $E_{2}^{p, q}=\left(R^{p} F\right)\left(R^{q} G\right)(-)$ and converges to $R^{p+q}(F G)(-)$, under the assumption that $\left(R^{p} F\right)(G I)=0$ for all injectives $I$ and $p>0$. In the case at hand, the functor $F$ is $\Phi_{*} \prod_{B P_{*} B P}(-)$ and the functor $G$ is $L_{n}$ (applied objectwise to the product category). Since $L_{n}$ preserves injectives [10, Corollary 2.4], the Grothendieck spectral sequence exists. Since $\Phi_{*}$ is exact and products of injectives are injective, we can use 
another Grothendieck spectral sequence argument to see that $R^{p} F=\Phi_{*} \prod_{B P_{*} B P}^{p}(-)$. Similarly, since $\Phi_{*}$ is exact and preserves injectives [10, Corollary 2.5], another Grothendieck spectral sequence argument shows that

$$
R^{p+q}(F G)(-)=R^{p+q}\left(\prod_{E(n)_{*} E(n)} \Phi_{*}\right)(-)=\prod_{E(n)_{*} E(n)}^{p+q} \Phi_{*}(-),
$$

completing the proof.

This proposition allows us to compute some products of $E(n)_{*} E(n)$-comodules. For example, we have

$$
\prod_{E(n)_{*} E(n)} \Sigma^{\alpha} E(n)_{*} \cong E(n)_{*} \otimes_{B P_{*}} \prod \Sigma^{\alpha} B P_{*},
$$

as long as the $\alpha$ are bounded below. To see this, use the fact that $B P_{*}$ is $L_{n}$-local [10], Theorem 6.2, and Theorem 6.3.

In fact, we have

$$
\prod_{E(n)_{*} E(n)}^{s} \Sigma^{\alpha} E(n)_{*}=0
$$

for $0<s<n$ and

$$
\prod_{E(n)_{*} E(n)}^{s} \Sigma^{\alpha} E(n)_{*} \cong \Phi_{*} \prod_{B P_{*} B P}^{s-n} \Sigma^{\alpha} B P_{*} /\left(p^{\infty}, v_{1}^{\infty}, \ldots, v_{n}^{\infty}\right)
$$

for $s \geq n$, again under the hypothesis that the $\alpha$ are bounded below. This follows from the spectral sequence of Theorem 6.3 and the fact [10] that $L_{n}^{q} B P_{*}=0$ except when $q=0$ and $q=n$, where

$$
L_{n}^{0} B P_{*}=B P_{*} \text { and } L_{n}^{n} B P_{*}=B P_{*} /\left(p^{\infty}, v_{1}^{\infty}, \ldots, v_{n}^{\infty}\right) .
$$

Note that we do not know whether $\prod_{B P_{*} B P} \Sigma^{\alpha} B P_{*} /\left(p^{\infty}, v_{1}^{\infty}, \ldots, v_{n}^{\infty}\right)$ is all $v_{n}$-torsion or not, and therefore we do not know whether $\prod_{E(n)_{*} E(n)}^{n} \Sigma^{\alpha} E(n)_{*}$ is zero or not.

\section{REFERENCES}

1. J. F. Adams, Stable homotopy and generalised homology, Chicago Lectures in Mathematics (University of Chicago Press, 1974).

2. J. M. Boardman, Conditionally convergent spectral sequences, in Homotopy invariant algebraic structures (Baltimore 1998), Contemp. Math., vol. 239 (Amer. Math. Soc., Providence, RI, 1999), 49-84.

3. E. S. Devinatz, Morava modules and Brown-Comenetz duality, Amer. J. Math. 119 (1997), 741-770.

4. P. G. Goerss, The homology of homotopy inverse limits, J. Pure Appl. Algebra 111 (1996), 83-122.

5. M. Hovey, Bousfield localization functors and Hopkins' chromatic splitting conjecture, in The Čech Centennial (Boston, MA, 1993), Contemp. Math., vol. 181 (Amer. Math. Soc., Providence, RI, 1995), 225-250.

6. M. Hovey, Homotopy theory of comodules over a Hopf algebroid, in Homotopy theory: relations with algebraic geometry, group cohomology, and algebraic K-theory (Evanston, IL, 2002), Contemp. Math., vol. 346 (Amer. Math. Soc., Providence, RI, 2004), 261-304.

7. M. Hovey, J. H. Palmieri and N. P. Strickland, Axiomatic stable homotopy theory, Mem. Amer. Math. Soc. 128 (1997), 610. 
8. M. Hovey and N. P. Strickland, Morava $K$-theories and localisation, Mem. Amer. Math. Soc. 139 (1999).

9. M. Hovey and N. P. Strickland, Comodules and Landweber exact homology theories, Adv. Math. 192 (2005), 427-456.

10. M. Hovey and N. P. Strickland, Local cohomology of $B P_{*} B P$-comodules, Proc. London Math. Soc. (3) 90 (2005), 521-544.

11. D. C. Ravenel, Complex cobordism and stable homotopy groups of spheres, Pure and Applied Mathematics, vol. 121 (Academic Press 1986).

12. C. A. Weibel, An introduction to homological algebra (Cambridge University Press, 1994). 